\title{
Antibacterial potential of catechin of tea (Camellia sinensis) and its applications
}

\author{
Rahardiyan, D. \\ Faculty of Agriculture, De La Salle Catholic University, Kairagi Kombos Manado 95000 Indonesia
}

Article history:

Received: 5 May 2018

Received in revised form: 5

June 2018

Accepted: 22 June 2018

Available Online: 3 July 2018

Keywords:

Catechin,

Tea,

Green tea,

Antimicrobial agents

\section{DOI:}

https://doi.org/10.26656/fr.2017.3(1).097

\begin{abstract}
By reviewing various works of research by many experts on tea, this mini review elaborates the picture of catechins in tea, especially green tea, and their applications as potential antimicrobial agents. Tea originated in China as a drink with medicinal purposes and to be grew a worldwide beverage. Green tea undergoes minimum oxidation process compared to black tea, thus it may contain the same pharmacological bioactive components, but in different concentrations, except for theaflavins and thearubigens that are mainly found in black tea. Phytochemical screening of green tea leaves revealed various polyphenols, mainly catechins, which play multiple essential roles in plant physiology and have potential health properties and benefits on human health, such as antioxidants, as well as anti-allergic, anti-inflammatory, anticancer, antihypertensive, and as antimicrobial agents. Green tea polyphenolic EGCG has the capability to cross-link with many proteins which grants it a wide range of antimicrobial activities possibly by damaging microbial cytoplasmic lipids and proteins. The high concentrations of catechins in tea (Camellia sinensis) were reported to have antimicrobial activities against many pathogens including Listeria monocytogenes, a gram-positive bacterium known to be isolated from red meat, therefore making tea catechin an effective antimicrobial agent. In a study model applying up to $1000 \mathrm{ppm}$ tea catechin extract to a biodegradable film, it proved beneficial for home-use wraps for meat, poultry, and seafood, thus expressing the strong potential of tea catechin as an organic antimicrobial agent.
\end{abstract}

\section{Tea, its antibacterial quality, and its applications}

The habit of drinking tea originated from China with medicinal purposes, possibly dating back to $2700 \mathrm{BC}$, and later became a popular beverage worldwide. It began with the hygienic practice of boiling drinking water which was further made more palatable by the addition of fragrance leaves from the tea plant (Taylor et al., 2005). In the following years, a huge interest grew towards its extensive health benefits (Wang et al., 2011).

Terminologically, Taylor et al. (2005) noted the confusion in the multiple definitions given due to the varieties and to the broad definition of 'tea' as an aqueous infusion of any herbs (as 'mint tea', 'rooibos tea' etc.), deriving from the French expression of tisane. The word 'tea' as used by Taylor et al. (2005) was then solely understood as the description of the leaves of a shrub, Camelia sinensis; whose leaves of this shrub are picked fresh as "two and a bud" (known as the 'flush'), further processed (macerated and heat-dried leading to green tea, or fermented and heat dried leading to black tea), and finally infused in boiling water. Gramza et al. (2005) elaborated that tea leaves are obtained from shrubs or trees of the Theaceae family (of the Camelia species). Tea leaves are dark green in color with a shiny surface, opposites and round, while the flowers are large and red, white and pink in color; and the fruits, small and brown. The major varieties are the Chinese tea shrub (Camelia sinensis) and the Indian tea tree (Camelia assamica).

Food-borne microbial pathogens contaminating food has been a concern for man for a long time. Thus, mankind's attempt at controlling microbial food contamination has also been a long journey; from old school methods such as heat-processing and coldprocessing to chemically induced antimicrobial agents. Consumer awareness, concerns over the potential risk of synthetic food additives on human health and also the pressing concerns for the freshness of food has lad to a renewed interest in the exploration of more organic and natural methods of food preservation (Nanasombat and Lohasupthawee, 2005; Si et al., 2006). The activities of tea polyphenols, green tea in particular, according to $\mathrm{Si}$ et al. (2006) have the ability to strongly inhibit the growth of major food-borne pathogens. 
Zhao et al. (2001) reported that the MIC (Minimum Inhibition Concentration) of Epigallocatechin-gallate (EGCg) of green tea towards Escherichia coli was more than $800 \mu \mathrm{g} / \mathrm{L}$ while towards Methicillin Susceptible Staphylococcus aureus (MSSA) and Methicillin Resistant $S$. aureus (MRSA) $(100 \mu \mathrm{g} / \mathrm{L})$, making evident that the EGCg of green tea is an effective antibacterial agent. Moreover, Cho et al. (2012) indicated in a study that from a dental health standpoint, the green tea polyphenols containing EGCg have the capacity to inhibit the growth of biofilm formation on human teeth, and evidently in the findings of Cho et al. (2012) green tea polyphenols were very effective against Lactobacillus spp., Streptococcus spp., S. aureus, Nisseria meningitidis, Escherichia coli and Candida albicans. Therefore, it can be concluded that green tea polyphenols may effectively be developed as antimicrobial agents against oral microorganisms, and have a great potential use as a mouthwash solution.

\section{Green tea vs. black tea}

Green tea according to Chakraborty and Chakraborti (2010) has various added values compared to black tea mainly due to the fact that green tea undergoes minimum oxidation processes since it is not "fermented", unlike black tea, thus preventing the bioactive components of green tea from undergoing further oxidation processes. Concurring with Chakraborty and Chakraborti (2010), Hamilton-Miller (1995) also reviewed that green tea, popular in the Far East, differs from the western black tea in terms of the oxidation process (termed as 'fermentation') that the latter undergoes. Taylor et al. (2005) further emphasized that black tea derived from the 'fermentation' process of the flush before heatdrying, thus containing many of the same pharmacologically active components as green tea (that was only macerated before heat-drying), but at lower concentrations. Black tea otherwise has higher molecular weight oxidation products, as theaflavins and thearubigens that are not found in green tea.

\section{Polyphenols of tea}

Phytochemical screening of tea revealed the presence of various compounds such as alkaloids, saponins, tannins, catechins and many other polyphenols (Archana and Abraham, 2011). Polyphenols are secondary metabolites produced by the tea plant, which play multiple essential roles in plant physiology and have potential health properties and benefits for human health, such as antioxidants, as well as anti-allergic, antiinflammatory, anticancer, antihypertensive, and antimicrobial agents (Koech et al., 2013).

Polyphenols in tea leaves can be categorized into 3 groups: catechins, theaflavins, and thearubigens. While both green and black tea contain similar quantities of flavonoids, their chemical structure differs. Green tea would appear to have more simple flavonoids - such as catechin, than black tea in which due to the 'fermentation' (oxidation) process these simple polyphenols are to be transformed into more complex flavonoids (namely the theaflavins and thearubigens). Furthermore, other oxidation processes of theses flavonoids would result in tannins which were classified into two groups of hydrolysable tannins (phenolic acids and gallic acid esterified polyols), and a group of nonhydrolysable (condensed) tannins, composed of mainly polymers of elementary flavonoid particles (Gramza et al., 2005). According to Reygaert (2014), the variety of tea based on their different processing methods result in variations in their catechin contents, with green tea containing the most, followed by oolong tea and finally black tea with the least. Besides not going through the oxidation ('fermentation') process, the initial steaming process of the macerated green tea leaves destroys the enzyme polyphenol oxidase, thus the polyphenols are protected from undergoing oxidation.

Total tea polyphenols of tea which were processed from germ plasms of different origin, investigated by Koech et al. (2014) using the Folin-Ciocalteu phenol reagent method were significantly different $(\mathrm{P}<0.05)$. Kenyan green tea germ plasm had higher total phenols ranging from $20.2 \%$ to $24.4 \%$ compared to Chinese and Japanese germ plasms which had $16.7 \%$ and $18.5 \%$ respectively. As with total catechins, which were investigated using a modified high-pressure liquid chromatography (HPLC) assay, Kenyan green tea germ plasm was higher than the Chinese and Japanese germ plasms $(\mathrm{P}<0.05)$. This difference was not significant for total theaflavins and thearubigens $(\mathrm{P}<0.05)$ of black tea from germ plasms of these origins.

\section{Catechins of tea and their detection of tea catechins}

It can be drawn from previous studies that, tea catechins may present at concentrations up to $1 \mathrm{mg} / \mathrm{mL}$ per cup of tea (specifically green tea) and can be classified into 4 major groups: Epicatechin (EC), Epicatechin-3-Gallate (ECG), Epigallocatechin (EGC), and Epigallocatechin-3-Gallate (EGCG). EGCG is known to be the most abundant in green tea, adding up to approximately $59 \%$ of total catechins, followed by EGC at $19 \%$, ECG at $13.6 \%$ and the finally EC at $6.4 \%$ (Hamilton-Miller, 1995; Archana and Abraham, 2011; Reygaert, 2014). Indeed, EGCG is not found in any other plants and is the major catechin of the flush, the growing point of the tea plant, consisting of the buds and 
immature leaves that are picked for processing (Hamilton-Miller, 1995).

Saito et al. (2006) developed an analytical HPLC detection method for detecting and quantifying Caffein, Catechin, Epicatechin, and Epigallocatechin-gallate in green tea (Camellia sinensis). The method relies on an RP-18 Column and a mobile phase composed of water, acetonitrile, methanol, ethyl acetate and glacial acetic acid at a ratio of 89:6:1:3:1 ( v/v/v/v/v) for simultaneous determination and quantifications. The method was used to simultaneously detect and quantify caffeine, catechin, epicatechin, and epigallocatechin-gallate of Brazilian spring, autumn and summer harvests of green tea in comparison to Brazilian black tea, Japanese and Chinese green tea and to two standardized green tea extracts. This method developed by Saito et al. (2006) proved statistically sound and adequate for determinations and quantification of the major components of tea.

\section{Catechins and their antimicrobial properties}

Microbial resistance has been described by Koech et al. (2013) to have become an increasing global problem, consequently making the search for novel potent antimicrobial agents a pressing matter. Earlier studies reported the prophylactic properties of tea against typhoid, as an army surgeon recommended tea as fluids for the army's drinking bottle (Hamilton-Miller, 1995). Along the years, further studies have suggested the much useful antimicrobial effects of tea polyphenols towards not only intestinal pathogens but also towards a wider spectrum of microorganisms including bacterial, fungal and viral pathogens (Zhao et al., 2013). Furthermore, Zhao et al. (2013) implied that green tea polyphenolic EGCG has the capability to cross-link with many proteins, which grants it a wide range of antimicrobial activities possibly by damaging microbial cytoplasmic lipids and proteins. Mahmoud et al. (2013) explained that the main antibacterial mechanism of EGCG is attributed to its capacity to directly attach itself to the peptidoglycan layer of bacterial cell walls, causing damages to the cross-linking peptides and thus damaging the cell walls. While this effect is not limited by species selectivity, it may contribute to the differences reported by various researches with regards to the minimum inhibition concentration (MIC). Mahmoud et al. (2013) also mentioned that the accumulation of autolysin enzymes within the cell walls that inhibits the cell separation during growth and formation of defective multicellular forms might another factor contributing to the variances of MIC. Concurring, Zhao et al. (2013) reports that EGCG exerts these antibacterial activities partly by damaging bacterial cytoplasmic lipids, membrane proteins or cytoplasmic enzymes such as gelatinase, protein tyrosine phosphatase, dihydrofolate reductase, DNA gyrase, etc. Therefore, EGCG also exhibits the capacity to impair bacterial resistance to antibiotics and thereby render bacteria more sensitive to various anti-bacterial agents.

Bakkir et al. (2011) studied the antimicrobial effects of green tea extract in vivo by subcutaneously inoculating four rabbits with methicillin resistant $S$. aureus (MSRA), the degree of swelling and necrosis was examined, the presence of green tea was associated with a reduction in swelling and in the severity of necrosis. Investigations on antimicrobial effects of aqueous green tea extracts on isolated $S$. aureus, $S$. pyogenes and $P$. mirabilis of the impetigo disease in children were conducted by Al-Kayali et al. (2011). Effects of various antibiotics were compared to the $10 \%$ aqueous green tea extract, and the study revealed that $10 \%$ aqueous green tea extract was just as effective against the antibiotic resistant $S$. aureus, $S$. pyogenes and $P$. mirabilis.

\section{The extraction process}

In general there are various methods for extracting phytochemicals, depending on the texture and moisture of a collected plant tissue sample, as well as on the compound properties to be extracted and isolated. A classic extraction method is by continuous soxhlet extraction with consecutive different solvents, starting with ether then petroleum ether and chloroform (to separate lipids and terpenoids) and alcohol and ethyl acetate are for the more polar compounds (Harborne, 1984). In order to identify and isolate phenolic compounds in general, Harborne (1984) recommended the best method for phenol identification and isolation would be by thin layer chromatography. The extraction process of tea catechin as well as with all tea polyphenols is commonly done using hot water and organic solvent, by which the yield of solvent extraction is much dependant on the types of solvent whose properties vary in terms of polarity, extraction time, temperature, and especially on the physicochemical properties of the sample itself (Lee et al., 2013). Mbata et al. (2006) extracted tea compounds (Camellia sinensis) via the aqueous extraction method and the methanol extraction method. The aqueous method consists of a 2 day-long soaking in $35 \mathrm{~mL}$ distilled water in a $250 \mathrm{~mL}$ sterile conical flask, and the methanol extraction consists of a 6-hour Soxhlet extraction with $250 \mathrm{~mL}$ of $95 \%$ methanol, and finally concentrated by evaporation in a water bath at $100^{\circ} \mathrm{C}$.

Lee et al. (2013) refined the extractions conditions of antioxidants from green tea by using the response surface methodology (RSM). Using ultrasonic extraction, the 
extraction conditions of antioxidants with relatively low caffeine content in green tea were optimized. Lee et al. (2013) predicted the optimal conditions for the highest antioxidant activity and minimum caffeine level at $19.7 \%$ ethanol (26.4 mins extraction time and $24^{\circ} \mathrm{C}$ extraction temperature), where the experimental values were very close ( $20 \%$ ethanol, 26.0 mins extraction time and $24^{\circ} \mathrm{C}$ extraction temperature). Whilst in their findings, the ratio of (EGCg $+\mathrm{ECg}) / \mathrm{EGC}$ was the major contributor to green tea extract antioxidant activities.

\section{Catechins as a red meat preservative}

Aslam et al. (2000) implied that red meat is an abundant protein and nutrient source, where the surfaces of meat cuts support the growth of a large number of microorganisms, while in ground meat, not only surfaces but the grinding process itself may act as growth media as the latter would be a thorough inoculation mix. Therefore, fresh comminuted meat will invariably have higher numbers of microorganisms than noncomminuted meat such as steaks and cuts.

The high concentrations of catechins in tea (Camellia sinensis) were reported to have antimicrobial activity against many pathogens, while in an investigation by Tiwari et al. (2005), it was reported that acidic, base and neutral methanol extract fractions of Camellia japonica inhibit the growth of food-borne pathogens in microbiological media and food. Green tea catechin extracts, according to Lee et al. (2013), have been implemented in functional food for their health benefits as well as their antioxidant and antimicrobial activities to increase shelf life. For example, catechin extracts have been added to cereal products, cakes, biscuits, ice cream, instant noodles, fried snacks, sausages and soft drinks. Mbata et al. (2006) tested antimicrobial activities of aqueous and methanol extracts of green tea (Camellia sinensis) on L. monocytogenes using agar-gel diffusion, paper disk diffusion, and microbroth dilution techniques. The extracts produced inhibition zones at a range of 10.0-20.1 mm against $L$. monocytogenes, thus indicating that the extracts are effective antimicrobial agents, while the minimum inhibitory concentration (MIC) of methanol and water extract were 0.26 and $0.68 \mathrm{mg} / \mathrm{mL}$, respectively. The idea of testing on L. monocytogenes is uniquely due to the fact that L. monocytogenes is not only a grampositive bacterium that is salt resistant and highly adaptive but also isolated from silos, vegetable, dairy products, most ready-to-eat foods, and especially from red meat.

One of the most promising modern preservation technologies for preserving raw or processed meat nowadays is the use of edible film coated with substances containing antimicrobial properties since they provide a good barrier against pathogens and spoilage microorganisms (Sanchez-Ortega et al., 2014). Alvarez et al. (2014) also regarded the promising advantages of edible films as carriers of antimicrobial agents to ensure food safety and quality, further it is explained that pathogenesis in food bacteria is related to a communication mechanism at the cellular level called quorum sensing (QS). Therefore Alvarez et al. (2014) researched the application of pectin films enriched with phyto compounds with anti-quorum sensing properties and demonstrated that Oregano Essential Oil (OEO) enriched Pectin Films were effective against E. coli O157: H7, S. aureus, and L. monocytogenes. Wu et al. (2010) applied catechin as a phytochemical with antimicrobial and antioxidant properties into a biodegradable film of PVA (polyvinyl alcohol) used as a wrap for fresh meat products such as red meat, poultry and seafood. Wu et al. (2010) had a study model of PVA -starch film that inhibited the growth of airborne microorganisms at a level of $1.000 \mathrm{ppm}$ catechins, and an optimum antioxidant activity towards cooking oil when $500 \mathrm{ppm}$ catechins were incorporated in the film. Thus the film developed by $\mathrm{Wu}$ et al. (2014), was beneficial as a home-use wrap for meat, poultry and seafood, which are considered as product susceptible to oxidation and microbial growth.

\section{Conclusion}

Many research on green tea phytochemicals evidently pointed that tea catechins play multiple essential roles in plant physiology, and have potential health properties and benefits on human health, such as antioxidants, anti-allergic, anti-inflammatory, anticancer, antihypertensive, and as well as antimicrobial agents. Polyphenolic EGCG of green tea apparently has shown the capacity to cross-link with many proteins, and consequently also displays a wide range of antimicrobial activities, in which the mechanism is possibly by damaging microbial cytoplasmic lipids and proteins. The extraction process of green tea catechin begins by the classic continuous Soxhlet extraction with different solvents, starting with ether then petroleum ether and chloroform (to separate lipids and terpenoids), and further refined by thin layer chromatography. Furthermore, the extraction process may be optimized by response surface methodology (RSM). Despite all the intricate methods of catechin extraction, a simple crude aqueous extraction method and methanol extraction method was sufficient to extract a high concentration of catechin available for applications as an antimicrobial agent. High concentrations of catechins in tea was 
reported to have antimicrobial activity against many pathogens including L. monocytogenes a gram-positive bacterium is known to be isolated from red meat, therefore making tea catechin an effective antimicrobial agent. A study model applying up to $1000 \mathrm{ppm}$ tea catechin extract to biodegradable film proved beneficial for home-use wraps for meat, poultry, and seafood. Therefore the prospect of the application of green tea catechin as an antimicrobial agent in an ediblebiodegradable film has shown its credence to future implementations in the fight against food-borne pathogens and to the future of food packaging developments.

\section{Acknowledgements}

The author would like to place a special acknowledgement and appreciation to Mrs. Virginia Le Bihan for providing support by the profound proofreading and excellent language corrections.

\section{References}

Al-Kayali, K.K., Razooqi, B.M. and Mtaab, A.S. (2011). Antibacterial Activity of Aqueous Extract of Green Tea on Bacteria Isolated from Children With Impetigo. Diyala Journal of Medicine, 1(1), 37-43.

Alvarez, M.V., Ortega-Ramirez, L.A., GutierrezPacheco, M.M., Bernal-Mercado, A.T., RodriguezGarcia, I., Gonzalez-Aguilar, G.A., Ponce, A., Moreira, M.D.R., Roura, S.I. and Ayala-Zavala, J.F. (2014). Oregano Essential Oil - Pectin Edible Films as Anti-Quorum Sensing and Food Antimicrobial Agents. Frontiers in Microbiology, 5(699), 1-7. https://doi.org/10.3389/fmicb.2014.00699

Archana, S. and Abraham, J. (2011). Comparative Analysis of Antimicrobial Activity of Leaf Extract From Fresh Green Tea, Commercial Green Tea and Black Tea on Pathogens. Journal of Applied Pharmaceutical Sciences, 1(8), 149-152.

Aslam, A., Mariam, I., Haq, I. and Ali, S. (2000). Microbiology of Raw Meat. Pakistani Journal of Biological Sciences, 3(8), 1341-1342. https:// doi.org/10.3923/pjbs.2000.1341.1342

Bakkir, L.K., Yassen, R.T. and Mustaffa, R.M. (2011). In Vitro and In Vivo Study of Green Tea and Black Tea Antimicrobial Activity on Methicillin Resistant Staphylococcus aureus. Basrah Journal of Veterinary Science, 10(2), 1-12.

Cho, Y.S., Oh, J.J. and Oh, K. H. (2010). Antimicrobial Activity and Biofilm Formation Inhibition of Green Tea Polyphenols on Human Teeth. Biotechnology and Bioprocess Engineering, 15(2), 359-364. https:// doi.org/10.1007/s12257-009-0195-8
Gramza, A., Korczak, J. and Amarowicz, R. (2005). Tea polyphenols - Their Antioxidant Properties and Biological Activity - A Review. Polish Journal of Food Nutrition Science, 14/55(3), 219-235.

Hamilton-Miller, J.M.T. (1995). Antimicrobial Properties of Tea (Camellia sinensis L.) Minireview. Antimicrobial Agents and Chemotherapy, 39(11), 2375-2377. https:// doi.org/10.1128/AAC.39.11.2375

Harborne, J.B. (1984). Phytochemical Method, $2^{\text {nd }}$ ed. London: Chapman and Hall Ltd. https:// doi.org/10.1007/978-94-009-5570-7

Koech, K.R., Wachira, F.N., Ngure, R.M., Wanyoko, J.K., Bii, C.C., Karori, S.M. and Kerio, L.C. (2013). Antimicrobial, Synergistic and Antioxidant Activities of Tea Polyphenols. In Méndez-Vilas, A. (Ed.) Microbial Pathogens and Strategies for Combating Them: Science, Technology and Education. Vol. 2, p. 971 - 981. Spain: Formatex Research Center

Koech, K.R., Wachira, F.N., Ngure, R.M., Wanyoko, J.K., Bii, C.C., Karori, S.M. and Kerio, L.C. (2014). Antioxidant, Antimicrobial and Synergistic Activities of Tea Polyphenols. African Crop Science Journal, 22(4), 837-846. https://doi.org/10.1016/ j.ijid.2014.03.631

Lee, L.S., Lee, N., Kim, Y.H., Lee, C.H., Hong, S.P., Jeon, Y.W. and Kim, Y.E. (2013). Optimization of Ultrasonic Extraction of Phenolic Antioxidants from Green Tea Using Response Surface Methodology. Molecules, 18, 13530-13545. https:// doi.org/10.3390/molecules 181113530

Mahmoud, M., Alkhaleefah, F. and Sheriff, D.M. (2013). Antimicrobial Effects of Epi-Gallo-Catechin-Gallate and Epicatechins of Green tea on Planktonic and Biofilm Forms of Staphylococcus aureus, Including MRSA. Nature and Science, 11(6), 70-79.

Mbata, T.L., Debiao, L.U. and Saikia, A. (2006). Antibacterial Activity of The Crude Extract of Chinese Green Tea (Camellia sinensis) on Listeria monocytogenes. African Journal of Biotechnology, 7, 1571-1573.

Nanasombat, S. and Lohasupthawee, P. (2005). Antimicrobial Activity of Crude Ethanolic Extracts and Essential Oils of Spice Against Salmonellae And Other Enterobacteria. KMITL Science and Technology Journal, 5(2), 527-538.

Reygaert, W.C. (2014). The Antimicrobial Possibilities of Green Tea. Frontiers in Microbiology, 5(434), 18. https://doi.org/10.3389/fmicb.2014.00434

Sanchez-Ortega, I., Garcia-Almendarez, B.E., SantosLopez, E.M., Amaro-Reyes, A., Barbosa-Corona, 
J.E. and Regalado, C. (2014). Antimicrobial Edible Films and Coating For Meat And Meat Products Preservation - A Review Article. The Scientific World Journal, 2014, 1-18. https:// doi.org/10.1155/2014/248935

Si, W., Gong, J., Tsao, R., Kalab, M., Yang, R. and Yin, Y. (2006). Bioassay-guided Purification and Identification of Antimicrobial Components in Chinese Green Tea Extract. Journal of Chromatography A, 1125(2), 204-210. https:// doi.org/10.1016/j.chroma.2006.05.061

Taylor, P.W., Hamilton-Miller, J.M.T. and Stapleton, P.D. (2005). Antimicrobial Properties of Green Tea Catechins. Food Science and Technology Bulletin, 2, 71-82. https://doi.org/10.1616/1476-2137.14184

Tiwari, R.P., Bharti, S.K., Kaur, H.D., Dikshit, R.P. and Hoondal, G.S. (2005). Synergistic Antimicrobial Activity of Tea and Antibiotics. The Indian Journal of Medical Research, 122(1), 80-84.

Wang, Y.F., Shao, S.H., Xu, P., Yang, X.Q. and Qian, L.S. (2011). Catechin - Enriched Green Tea Extract as A Safe and Effective Agent for Antimicrobial and Anti-inflammatory Treatment. African Journal of Pharmacy and Pharmacology, 5(12), 1452-1461. https://doi.org/10.5897/AJPP11.164

Zhao, W.H., Hu, Z.Q., Okubo, S., Hara, Y. and Shimamura, T. (2001). Mechanism of Synergy Between Epigallocatechin Gallate and $\beta$-Lactams Against Methicillin Resistant Staphylococcus aureus. Antimicrobial Agents and Chemotherapy, 45 (6), 1737-1742. https://doi.org/10.1128/ AAC.45.6.1737-1742.2001

Zhao, L., Li, W., Zhu, S., Tsai, S., Li, J., Tracey, K. J., Wang, P., Fan, S., Sama, A.E. and Wang, H. (2013). Green Tea Catechin Quenches the Fluorescence of Bacterial-Conjugated Alexa Fluor Dyes. Inflammation and Allergy-Drug Targets, 12(5), 308 -314 . https:// doi.org/10.2174/18715281113129990057 